\title{
Distributed Behavior Model Orchestration in Cognitive Internet of Things Solution
}

\author{
Chung-Sheng $\mathrm{Li}^{1}$, Frederica Darema ${ }^{2}$, and Victor Chang ${ }^{3}$ \\ 1. Accenture Operations, 50 West San Fernando St, San Jose 95113, USA \\ 2. Air Force Office of Scientific Research, Arlington, VA, USA \\ 3. Independent Researcher, Southampton, $U K$ \\ csli@ieee.org; frederica.darema@us.af.mil; ic.victor.chang@gmail.com
}

\begin{abstract}
Keywords: Internet of Things, CIoT, Smarter Planet, Behavior Models, Cognitive Computing, World Models, Smart Grid, Fog Computing, Edge Computing, Progressive Representation, DDDAS, Dynamic Data Driven Applications Systems, Infosymbiotic Systems, Autonomy.

Abstract: $\quad$ The introduction of pervasive and ubiquitous instrumentation within Internet of Things (IoT) leads to unprecedented real-time visibility (instrumentation), optimization and fault-tolerance of the power grid, traffic, transportation, water, oil \& gas, to give some examples. Interconnecting those distinct physical, people, and business worlds through ubiquitous instrumentation, even though still in its embryonic stage, has the potential to create intelligent IoT solutions that are much greener, more efficient, comfortable, and safer. An essential new direction to materialize this potential is to develop comprehensive models of such systems dynamically interacting with the instrumentation in a feed-back control loop. We describe here opportunities in applying cognitive computing on interconnected and instrumented worlds (Cognitive Internet of Things - CIoT) and call out the system-of-systems trend among distinct but interdependent worlds, and Dynamic DataDriven Application System (DDDAS)-based methods for advanced understanding, analysis, and real-time decision support capabilities with the accuracy of full-scale models.
\end{abstract}

\section{INTRODUCTION}

The rapid adoption of Internet of Things (IoT) together with unprecedented bandwidths and computational power in instrumentation devices have already produced ground-breaking realtime or near real-time visibility and analysis across a broad spectrum of system environments [Atzori et al., 2010; Chang, 2017 a; Grandos, 2014; Gubbi et al., 2013, Karagiannis et al., 2015; Riggins et al., 2015; Shang et. al., 2015]. These capabilities enable instant access and transfer of information about a system or device in both natural and engineered systems as well as in consumer and industrial environemnts [Zhou and Chou, 2011, Wellbourne et al., 2009; Zhai et al., 2016]. A few examples include:

- personal environment (wearable devices and smart phones),

- surrounding environment (bodycam), 
- home environment (home security devices, appliances)

- power grid (eMeters, Power Management Units (PMUs), other sensor and actuator in the power distribution systems)

- traffic and transportation ( traffic sensors on cars, busses, trains, roads, traffic lights, railroads, aerial/Unmanned Aerial Vehicles (UAVs), and congestion control devices)

- structural health monitoring (bridges, buildings, vehicles, aerial platforms)

- water systems (distribution grids, asset management and preventive maintenance; ambient environments)

- oil \& gas (intelligent oil field)

Interconnecting these distinct natural, engineered, people, and business worlds (as shown in Fig. 1) through ubiquitous instrumentation, even though still in its embryonic stage, has the potential to create an environment that can be greener, safer, more efficient, and more comfortable. However, just a compedium and deluge of instrumentation data is insufficient

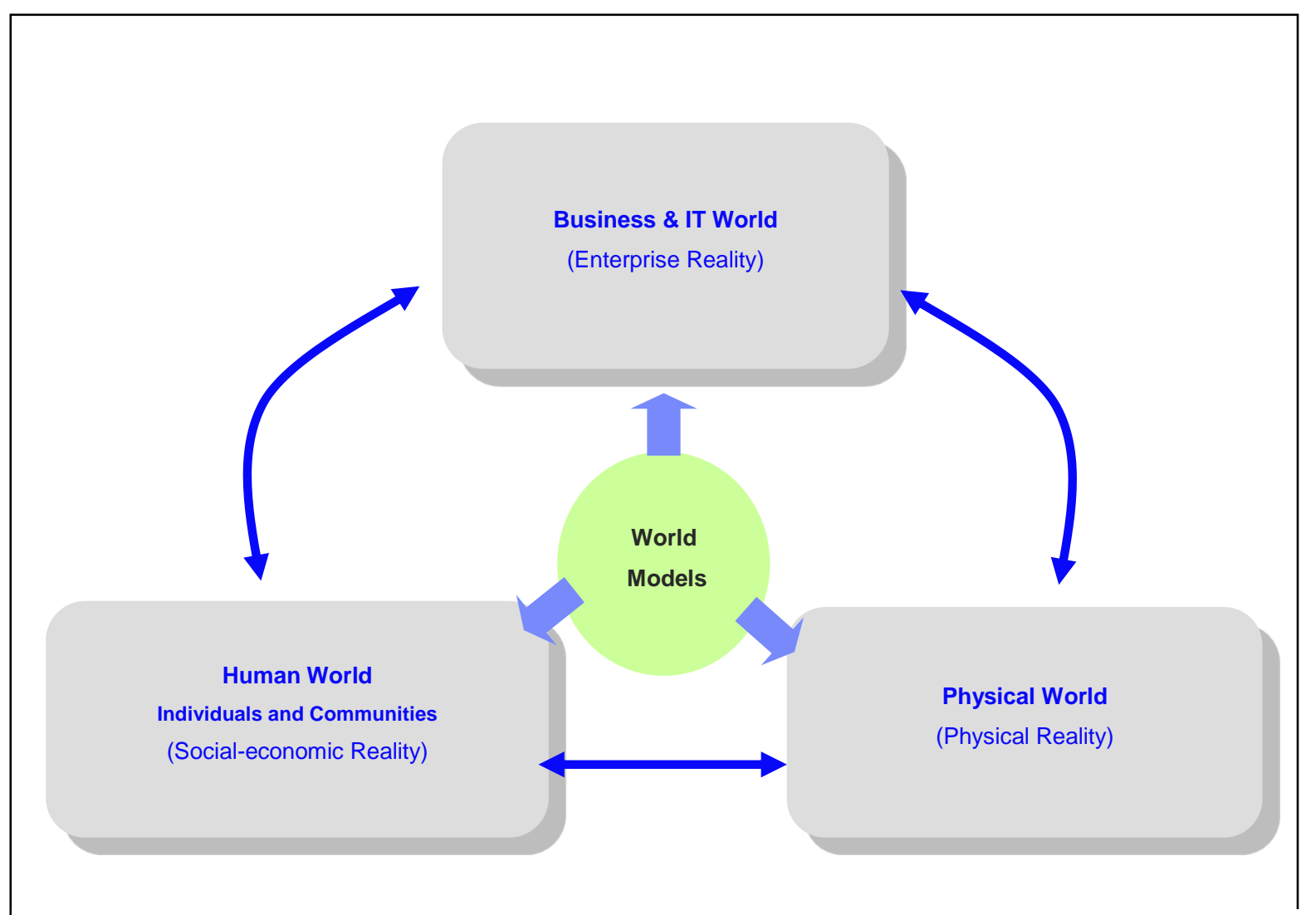

Figure 1: CIoT solutions will require interconnected and interdependent models representing the natural and engineered (physical) environment, business \& IT, and individuals \& communities. 
in achieving the ultimate objectives of a much better outcome for the system of interest and its environment [Li, 2005]. Cognitive representations (a.k.a. models) of these distinct natural, engineered, people, and business worlds are essential in understanding the complexity of these systems-of-systems, predicting their trajectory and controlling their actual evolution. Furthermore, cognitive representation for our emerging service and analytics can create accurate decision support capabilities when maneuvering through uncertain environments whose conditions are not known a priori. IoT combined with modeling is referred to as Cognitive IoT (CIoT) [Wu et al., 2014, Zaidi et al., 2015].

Rich multi-fidelity and multimodal modeling and instrumentation have become keys for enabling the above referenced capabiltiues for physical world systems (natural, engineered) and human systems. Beyond present notions of CIoT [Wu et al., 2014; Zaidi et al., 2015], new capabilties derived through Dynamic Data Driven Applications Systems (DDDAS)-based methods, whereby modeling is dynamically and synergistically integrated with instrumentation in a feedback control loop, are emerging [Darema 2000, 2005; Allaire et al. 2014, Bazilevs et al. 2012, 2013; Celik 2011, Celik et al. 2010]. The trend towards higher fidelity and (semi-)autonomy with humans in the loop is accelerating [Jense et al., 1997]. Related methods and opportunities include fusing multiple world models can be used to extract insights and capturing dynamic intelligent interactions in order to augment and enrich these in a CIoT environment as the connectivity and bandwidth could be severely challenged at times. This framework is validated through use cases such as load redistribution and disaster recovery for smart grid and structural health monitoring for intelligent aerial platforms (as for example discussed in [Darema 2000, 2005; Allaire et al. 2014, Bazilevs et al. 2012, 2013; Celik 2011, Celik et al. 2010]). interdependent world models. In addition, resilient IT infrastructures are essential for supporting the CIoT capabilities discussed above.

In this paper, we present a distributed framework for orchestrating both information and control flows within the instrumented environment based on interconnected cognitive models of these environments in order to achieve optimal outcome. The primary contribution of this paper is the proposed outcome-driven orchestration framework for complex CIoT systems and solutions. In contrast to the traditional orchestration approach where a detailed script is provided, the proposed approach specifies the desired outcome and orchestrates the 
information and control flows, using the behavior model of the CIoT system. The behavior model is incrementally refined based on the continuous learning on the actual outcome with respect to the desired outcome. Furthermore, the orchestration is executed based on progressive data representation and operations using a distributed model, which is essential

The rest of the paper is organized as follows: Section 2 describes the overall architecture for a cognitive IoT solution. A comprehensive landscape of cognitive models is described in Section 3. Section 4 discussed a number of use cases to illustrate the orchestration framework. The system and system software implications for designing a distributed orchestration framework are discussed in Section 5. Section 6 outlines the outcome-based distributed orchestration framework. A summary and potential future work is given in Section 7.

\section{COGNITIVE IOT SOLUTOIN ARCHITECTURE}

A Cognitive Internet of Things (CIoT) solution is a system with feedback control loop (or system of systems, since each individual component within this system could be a system by itself). Figure 2 shows the system view of such a closed-loop CIoT solution:

- Modeling \& Orchestration Platform

- Data \& Measurement Platform

- Control Platform

A CIoT solution inlcudes the real world itself - whether it is a smart grid, a smart building, a smart supply chain, or a smart water system. The data and measurement platform provides mechanisms to capture, comprehend, and learn from the real world (of varying levels of fidelity) to form the observed world. The observed world can be described by a set of world models.

Within the modeling and orchestration platform, cognitive representations (or models) integrating data captured from the instrumented real world enable interpolation or 
extrapolation to those areas of the phase-space ${ }^{1}$ of the system, where there are no modeling data or where the instrumentation data could be noisy, unavailable, or corrupted. And in other cases, these models allow generation of the most plausible hypotheses to explain the available information through deduction, induction, or abduction reasoning.

From these models, the possible outcomes are generated through simulation and/or predictive analysis. Based on the what-if analysis conducted by the models, a course of actions

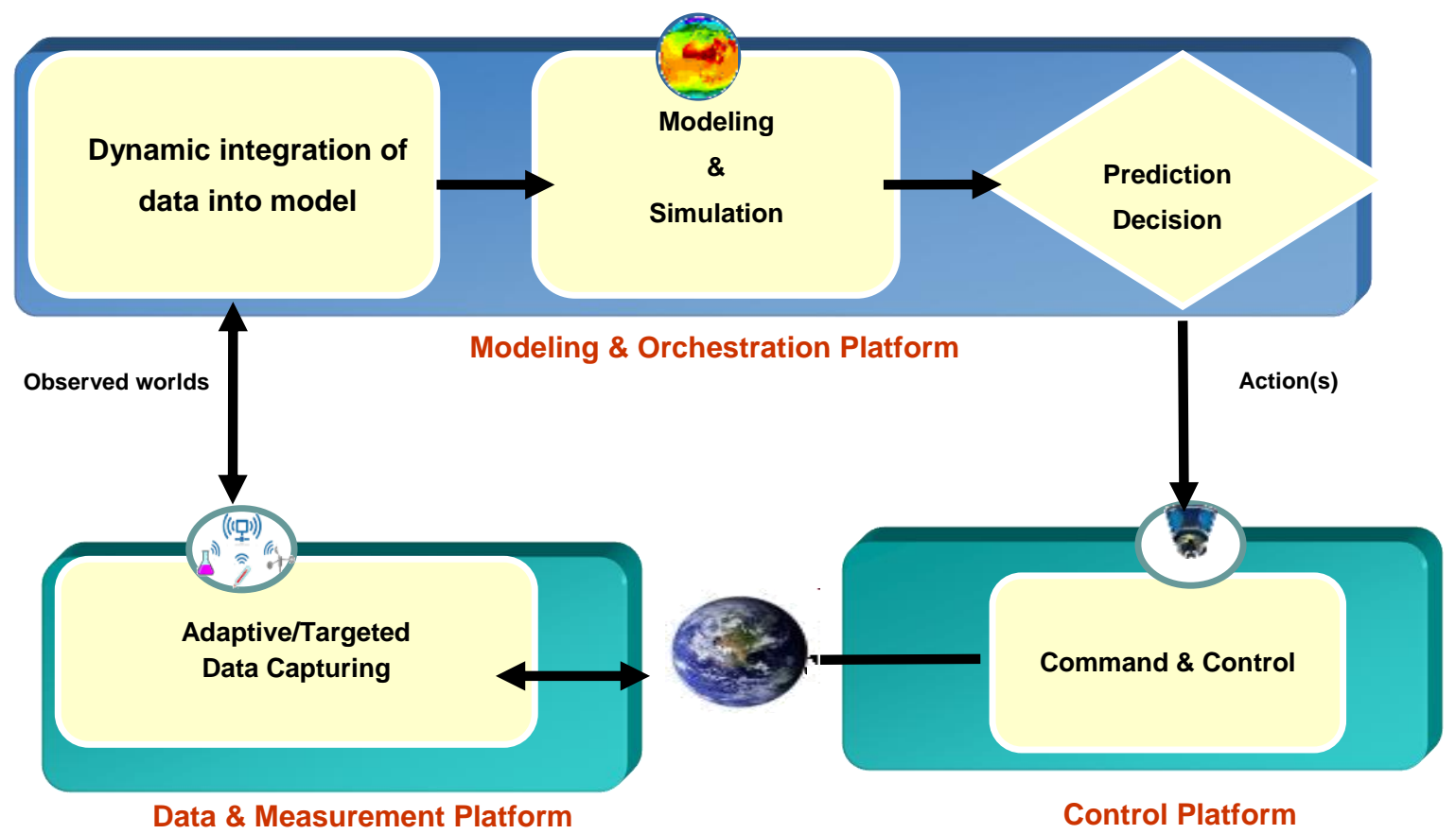

Figure 2: Interconnected platforms provide data dynamic capture \& integration into models, orchestration of behavioral models, and control for closed-loop prediction $\&$ response.

is then chosen to optimize the potential outcome. This decision is then implemented through

\footnotetext{
${ }^{1}$ phase space of a dynamical system is a space in which all possible states of a system are represented, with each possible state corresponding to one unique point in the phase space.
} 
the control platform to actuate the real world. The consequence in the real world, or the outcome, will be monitored through the data and measurement platform, and may result in more targeted data acquisition for more intelligent probing of the observed world, as well as adjustment on the course of actions.

This cognitive IoT solution in reality is an instance of the closed-loop control system and is similar to the $\mathrm{MAPE}^{2}$ loop of an autonomic system, with the exception that the role, influence and effects from human (individually or as a community) need to be included in the system.

A CIoT solution requires optimal or near optimal orchestration of the control flow and information flow. (The music notes of the orchestration really came from the behavior models, which dynamically integrate real-world information, conduct what-if analysis, and choose the course of actions that is likely to optimize the business outcome). Consequently, developing models at the behavior levels, is necessary in order to enable optimal orchestration of both information and control flows [Bates, 1995]. The behavioral models are continuously updated by the dynamic data inputs, either to speed-up the execution (through substituting parts of the computation in the model with the actual data) or to impart additional information into the model, (as it is quite often that the model does not accurately or fully captures the system). The output of the behavior model controls the instrumentation in order to either refine data acquisition to improve the model accuracy or actuates the controllers to effect an action (on the system or by the system) guided by a comprehensive model of the system. This category of systems was referred to as the Dynamic Data-Driven Application Systems (DDDAS), which are based on the Infosymbiotics ${ }^{3}$ paradigm [Darema 2000, 2005, 2006, 2010]. The kinds of

\footnotetext{
${ }^{2}$ MAPE (Monitoring-Analysis-Planning-Execution)

${ }^{3}$ The terms InfoSymbiotics or InfoSymbiotic Systems are used to denote DDDAS-based methods and capabilities
} 
models of interest span numeric and non-numeric, agent- based and graph models, as well as statistical models. Examples will be given in the next section.

\section{MODELS}

Cognitive representation (a.k.a. models) of the real world is the glue among data and measurement platform, modeling and orchestration platform, and the control platform. There are multiple abstraction levels of models of the real world. The most abstract level is at the

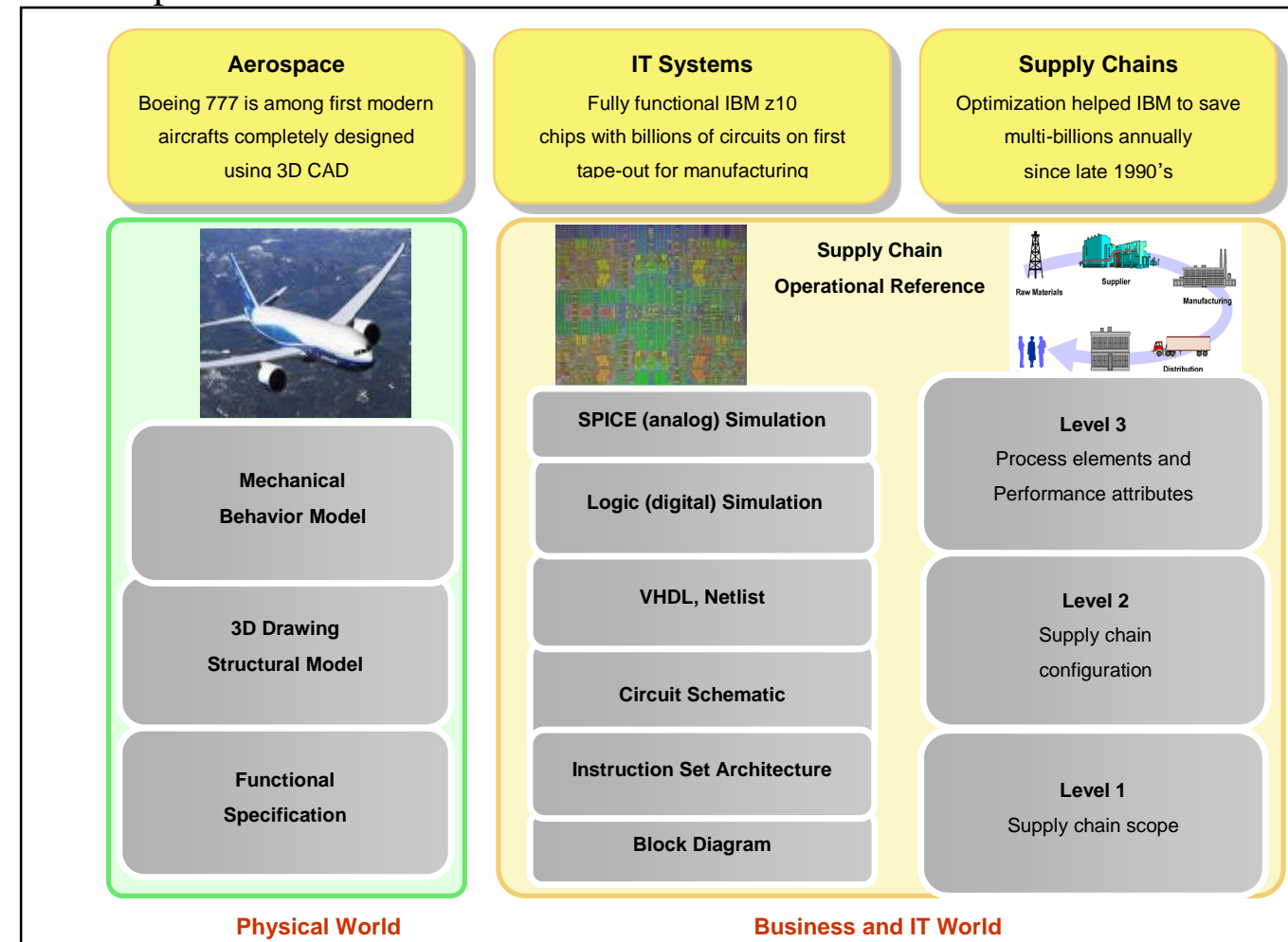

Figure 3: Examples of multiple abstraction levels of models of the world.

conceptual (theory or functional) level. Conceptual level models can be represented in the form of ontology, rules, or knowledge graph. Additional details are available at the structural level, which often captures the topological or spatial characteristics of the real world. Behavioral level models, which often describes the temporal or spatiotemporal evolution of a system, capture the most comprehensive aspects of the real world. Multiple levels of fidelity may be used in describing the system at hand at both the structural and the behavioral level. The evolution of the abstraction levels of the model typically starts at the conceptual and/or 
functional levels. The evolution from conceptual/functional models to behavior models in almost every domain in the past has improved outcomes (e.g. in business) while maintaining manageable complexity and uncertainty, as shown in Fig. 3.

During the development of Boeing 777, a dynamic behavior model of the aircraft was developed in addition to the traditional functional specification (at the function level) and 3D mechanical drawing (at the structural level). The dynamic behavior of the aircraft was evaluated within a simulation environment rather than going through numerous wind-tunnel testing [Abarbanel, 1996]. In a later section, we discuss new and more powerful methods (DDDAS-based) that not only allow optimal design of aircraft, but also use new modeling methods to enable optimized operational capabilities under dynamic conditions.

Additional examples include the development of the IBM z10 chip. The z10 development effort started with the functional specifications and the design of the instruction set architecture (ISA) - both at the functional level. Based on the functional specification, the VHDL specification (VHSIC Hardware Description Language) for the overall design was created. The circuit layout, which is a structural representation of the $\mathrm{z} 10$ chip, is then synthesized from the VHDL specification. Alternative structural representation, in the form of circuit schematic and netlist, is also synthesized from the VHDL specification. These VHDL and netlist representations of the z10 chip enable both logical and analog behavior of the z10 chip to be fully simulated within the simulation environment. The ability of fully capturing the IBM z10 system at the behavior level enabled the system to be entirely operational on the first tape-out of the chip [Lets et al., 2009].

Similarly, the enterprise supply chain can be specified at the functional (supply chain scope), structural (supply chain configuration), and behavioral (process elements and performance attributes) levels. The ability to perform process design and optimization of a supply chain at the behavior levels saves multi-billion dollars annually for some of the enterprises [Min, 2002; Celik et al., 2010].

In general, the entire CIoT spectrum really includes natural and engineered worlds, business and IT worlds, and the human worlds, and can be further divided into at least six domains: natural and engineered (compute intensive), embedded (SCADA related), cyber, enterprise, community, and individuals. 


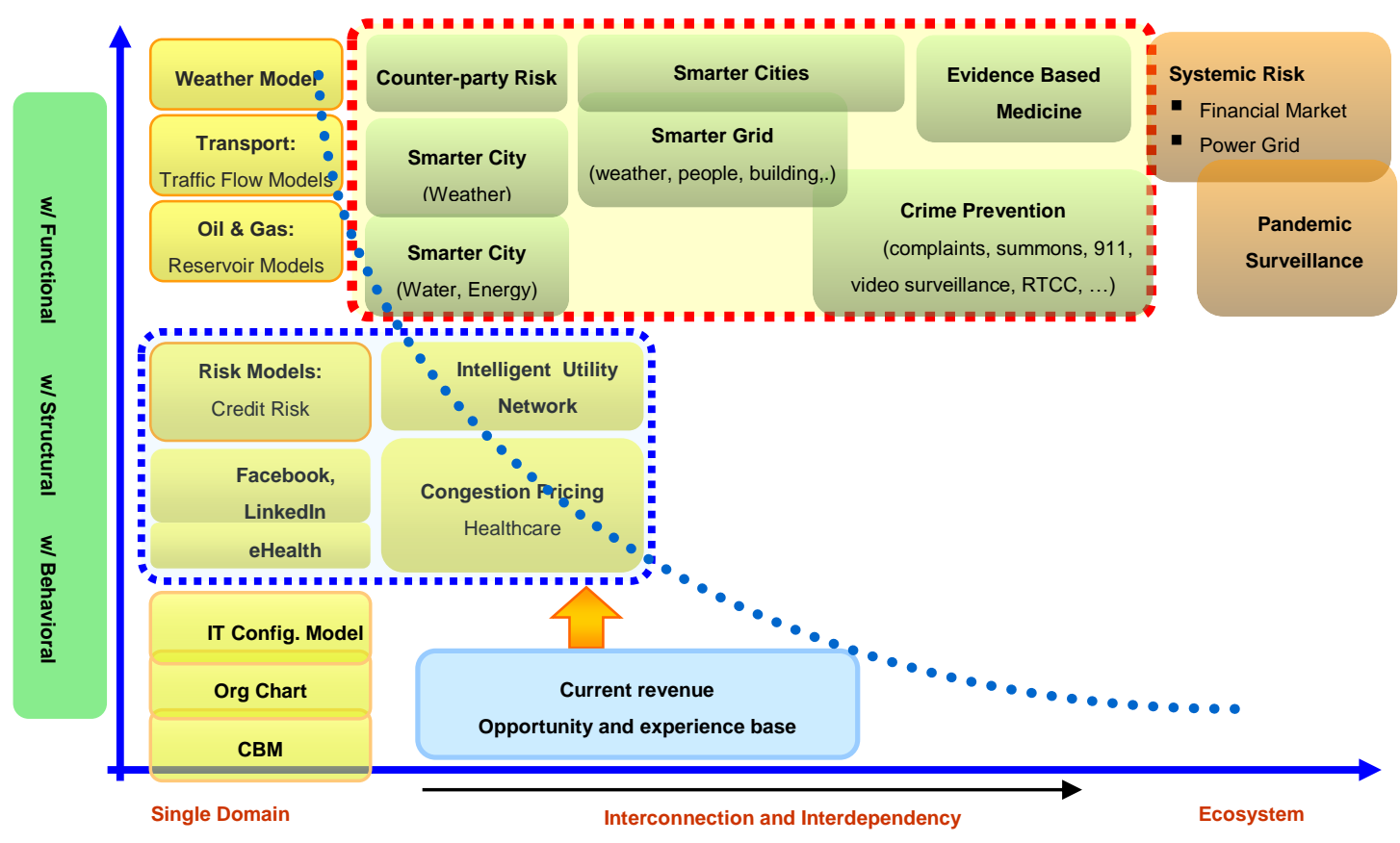

Figure 4: Future CIoT solutions will require interconnected and orchestrated measurements and models across multiple domains.

During the past few decades, cognitive models in each of these silos are evolving from functional to structural and now to behavioral level. In the foreseeable future, capturing and modeling the CIoT will happen at multiple levels of abstraction, multiple levels of fidelity, and from multiple vantage points.

In the enterprise domain, CBM (component business model) [Chesbrough, 2010] and industry framework captures the functional aspect of a business. Industry models (including data models, ontology models, knowledge graph models, process models, and service models) capture the structural levels of the business. Customer and workforce logistics and the enterprise risk models capture the behavioral levels of the business.

In the cyber area, ITIL [Canon, 2011] can be viewed as belonging to the functional level while IT configuration model belong to the structural level and workload and network traffic belong to the behavior level. 
In human communities, social networks (including Google+, LinkedIn and Facebook) capture the structural level of human relationships. Many of them evolve into capturing social or community behavior in real time during the recent past. From the individual (personal) side, individual profile belongs to the functional level while the purchase history belongs to the behavior level.

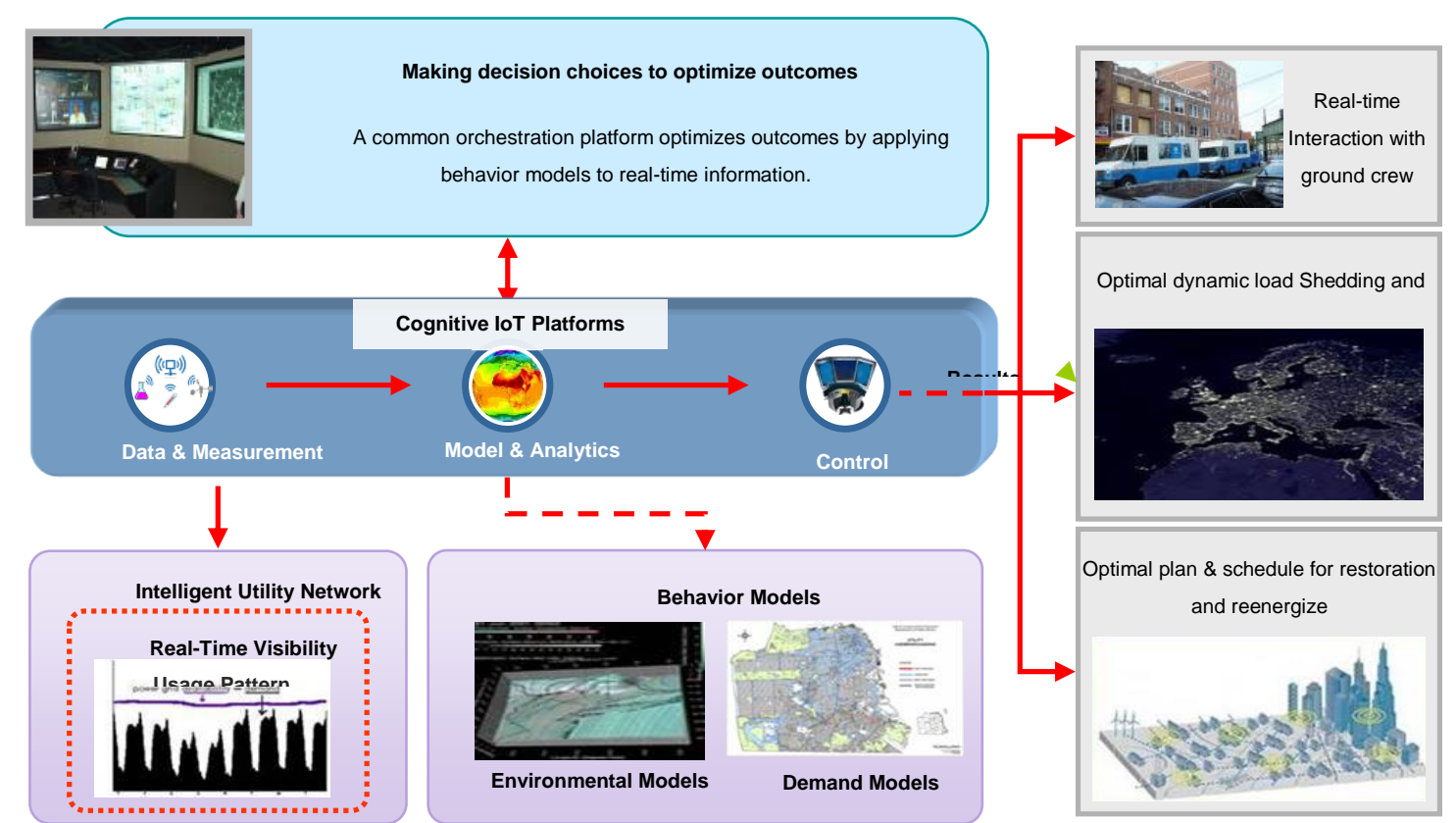

Figure 5: Smart Grid solutions continuously optimize the expected outcome dynamic data driven behavior models

The embedded systems domain includes examples such as instrumentation in the electric grid, transportation, dam, traffic lights, and manufacturing, where Supervisory Control And Data Acquisition (SCADA) systems [Boyer, 2009] are often deployed. This area is transforming itself at an extremely fast pace as increasingly more of such systems are connected to each other as well as to the internet, and through DDDAS-based models. This is also supporting a similar concept from Cheng et al. (2016) to allow intelligent manufacturing enabled by IoT.

New insight is established when analyzing the existing and future CIoT solutions in the context of an end-to-end ecosystem as opposed to a single domain, as shown in Fig. 4. Many of the existing CIoT solutions fall into the category of single domain, and leveraging only structural models for static analysis. Emerging opportunities - whether they are in the smart 
grid (power grid) or smart cities - often require integrating behavior models from two or more interdependent domains. New capabilities have been continuously demonstrated along these directions (to be shown in the next section), and are likely ready for industry deployment during the next 2-5 years.

\section{CASE STUDIES}

In this section, we will use a number of examples such as the smart grid and smart structures to illustrate the behavior-model-based orchestration in DDDAS-based CIoT solutions. The examples included here are only a subset of the capabilities that have been demonstrated in the recent past.

\section{(A) Energy related applications}

In a smart grid solution, intelligent utility networks (IUN) provide capabilities for real-time prediction of the onset of brown-outs or black-outs, and also provide optimized dynamic load management. The real-time instrumentation capability, often referred to as automatic meter reading (AMR) or automatic meter infrastructure (AMI), is based on measurements made by voltage amplitude phasor measurement unit (PMU) and other IoT sensors dynamically integrated with statistical and agent-based models. Dynamic load management within a Smart Grid solution activates mitigating actions prior to the onset of a brown-out or black-out to ensure differentiated support for critical and high-priority customers from multiple energysources, including renewables - such as wind, solar, and hydro, and energy storage which acts as a generation source [Celik 2013,2015]. This is the improvement based on enterprise storage solution from Chen et al., (2016).

Other scenarios include incorporating weather data and weather prediction models in dynamic data driven behavior models of the power-grid to provide continual optimization of load redistribution during peak demand period (such as during summer) or restoration of the grid infrastructure after a weather induced failure (integrated outage management). Emerging scenarios for supporting electric vehicles already present a new demand class and potentially a generation source (through the use of the batteries of these vehicles). Furthermore, demand- 
response management can mitigate demand during peak hours through incentives such as dynamic pricing plan.

With such capabilities, the utility companies will be able to provide much better assurance of the business outcome for their customers. The bottom line is to leverage the real-time visibility (instrumentation) and use DDDAS-based methods in order to build real-time behavioral models so that the business can optimize the expected outcome continuously.

Other Smart Grid related areas such as wind farms pose new challenges and require new CIoT capabilities. These CIoT capabilities enable optimized operation to mitigate effects of the wake across stacked turbines [Perez 2015]. They can also reduce the wear and tear of turbine rotors and provide an adaptive maintenance schedule rather than conducting maintenance on all turbines on the same regular schedule [Ding 2006]. This has a similar concept to Wang et al. (2016), which has Cloud-based production system with the fusion with IoT. Together with the fusion model, it blends Waste Electrical and Electronic Equipment to maximize outputs.

Accurate high resolution weather forecasts are central to predicting potential storm severity and its path. IBM Research's Deep Thunder [Gallagher, 2012] can provide high resolution forecasts for a 48-hour horizon for areas (in a given county) that are most likely to have outage events (and quantify the uncertainty level for the event to occur). Other examples are cited in Section 4(D) below, while additional capabilities for adverse weather prediction are discussed in Section $4(\mathrm{C})$. The ability to estimate the likelihood and severity of damage in different regions allows for predictive planning in stationing crews for early repairs. Such predictions can provide a basis for planning the deployment of repair crews and trucks in an anticipatory mode. Optimizing work orders and repair crews scheduling enables maximizing the business outcome in terms of the number of customers that are brought back online at the earliest possible opportunity. In addition, such capabilities allow optimized emergency response for any ensuing harm and damage to humans, buildings, and other infrastructure.

\section{(B) Structural Health Monitoring, Energy Efficiencies, and Decision Support}

Aerial platforms (both civilian and military, human-operated and UAVs, aerial and spacebased) during flight are subject to dynamic stresses accentuated by turbulence-induced forces. 
Such stresses as well as the aging of materials can result in structural damage, manifested as cracks, disbonding, delamination, or waviness. All these conditions can cause disastrous results (such as airplane crashing, as indeed happened in the past, in a case of aileron detachment). Additional sensor malfunction situations (such as pitot-tubes freezing) also result in catastrophic failures. DDDAS-based modeling have shown advanced capabilities: (1) detection of the onset of damage (crack creation) [Prudentio et al., 2013; Bazilevs et al. 2012], (2) predicting the propagation of the damage and potential impact [Allaire et al., 2014], (3) application of component- or system-level models for time dependent control through coordination of multiple actuators to mitigate the propagation of the damage [Bazilevs et al., 2012], (4) wing-level and aerial structure-wide structural health assessment, (5) multi-fidelity models dynamically driven by multiple levels of sensors (e.g. piezoelectric, optical, IR, ultrasonic) to assess platform health conditions in real-time [Bazilevs et al. 2012; Allaire et al., 2014], and (6) cognizant of environment (such as winds and wind-induced turbulence) to plan or re-plan in real-time to optimize the flight path and necessary maneuver to fulfill the mission. Such a decision support system leverages the accuracy of full-scale models during the optimization [Allaire et al., 2014]. It was demonstrated in [Varela 2013, 2014] that DDDAS-based methods enable detection and compensation for sensor failures; in this case, the output from a continually executing model of flight conditions is compared against the actual measurements from the pitot sensor. The model can take over in case of abrupt discrepancy with the measurement to allow time to readjust and switch over to other sensor modalities.

\section{(C) Atmospheric Contaminant Dispersion Monitoring}

Adverse atmospheric contaminant conditions, either naturally induced such as volcanic eruptions or man-made harmful chemical emissions (accidental, or intentional - such as in terrorist attacks), can have significantly disruptive effects. The impact of such events is further exacerbated due to the lack of comprehensive models for accurately attributing the origin and predicting the subsequent dispersion pattern of the contaminant. The consequence includes severe limitation on the effectiveness of the mitigating actions for evacuating humans (and animals) from affected areas, plan for decontamination, and any other needed emergency 
response actions. As an example, the April 2010 Eyafjallajokull volcanic eruption caused a 7day shutdown of much of northern Europe's air transportation system and affected many millions of passengers with an estimated cost between 1.5 and 2.5 billion of euro [Schmidt et al. 2011]. The traditional volcanic ash transport models over-predicted the ash spread in some areas and missed other areas where ash was actually dispersed, resulting in engine damages for aircraft flew over those regions. DDDAS-based modeling [Patra 2013; Stefanescu 2014], which also considered prevalent winds and dynamic incorporation of targeted satellite data (in this case used in a play-back fashion, as the referenced scientific work happened sometime after the actual event), showed improved accuracy in predicting the volcanic ash dispersion. The DDDAS-based modeling also demonstrated that ash presence in certain regions was missed due to misinterpretation of satellite images as it was difficult to distinguish between the reflections from ash particles and those from water vapor. Other DDDAS-based work [Peng et al. 2015, 2016] also provides improved contaminant plume tracking in an urban environment (the Manhattan cityscape) under air turbulence conditions from prevalent terraininduced winds. DDDAS-based methods were used to control where to send aerial mobile sensors, to regions of high-value data sampling (for example higher concentration of contaminant), while measuring winds and turbulence at the same time. Similar work on pollution transport was conducted by [Akcelik 2004, 2006] in open environments, and by [Carmichael 2003; Biswas 2014] on atmospheric pollution over the Pacific Ocean.

\section{(D) Adverse Atmospheric Weather and Space Weather}

Adverse weather such as tornadic activity affects safety of humans and property. In the United States, tornadoes cause multimillion dollar damage and loss of human lives each year. As an example, US Storm Prediction Center (SPC) reported a total of 1,285 tornado events during 2015 with 36 fatalities. Accurate and timely prediction of the onset of tornadic activity and of the path and extent of the tornado is of paramount importance to mitigate danger to human and animal lives as well as to improve preparedness and effective emergency response after tornadic events. Traditional modeling methods have now been replaced with DDDASbased modeling and instrumentation methods [Brotzge, 2004; Plale, 2005]. These new 
methods are able to predict tornadic activity indicators (such as the tornadic "hook" which often forecasts the onset of tornado) with much improved accuracy [Chang, $2017 \mathrm{~b}$ ].

In the outer space, space-weather affects the drag on satellites and impacts the accuracy of GPS. However, accurate modeling of the interdependency among various space weather phenomena in the ionotroposphere layer is very difficult as some aspects of the physics of these phenomena are not fully and accurately modeled yet. Factors in understanding spaceweather include the chemistry, the turbulence, and the thermal conductivity in the ionotroposphere. Among these three components, the thermal conductivity is most difficult to model accurately. DDDAS-based methods improved the model accuracy by leveraging the actual data to compensate for the model's deficiency [Ali 2015]. This resulted in more accurate modeling of the thermal conductivity component in the ionotropospheric layer, as manifested by the more accurate prediction of NO (nitrous oxide), neutral density, photoelectron heating efficiency, and the eddy diffusion coefficient.

\section{(E) Software Cybersecurity}

Opportunities for cyberattacks are increasing during the IoT era as wirelessly interconnected computing platforms and devices are proliferating. End-to-end encryption, due to its increased communication bandwidth and power consumption requirements, may be practically less applicable in some of the IoT environments. Randomization approaches such as instruction randomization, data randomization, and memory randomization also incur high overheads. DDDAS-based methods minimize the overhead by conducting the randomization adaptively at the application programming and execution environments levels. Through continuous monitoring and detection of increased vulnerability, randomized variations of sections or components of the software are created - including changing the programming language, changing the OS and middleware services, and changing the hardware resources. It was shown in [Hariri et al. 2015] that 4-way randomization guarantees zero probability of successful attack even for components with high probability ( $90 \%$ in the test case) of attack.

\section{(F) Intelligent Oil Field}


The petroleum industry has consistently driven the adoption of advanced modeling and instrumentation methods to optimize both upstream (oil/gas exploration) and downstream (oil/gas extraction) operations. To develop comprehensive oil/gas reservoir models, it is often required to interpolate low spatial resolution (but wide spatial coverage) seismic/sonic measurements with high resolution (but small spatial coverage) electrical conductivity (FMI) and radiation (gamma-ray, neutron, and proton) measurements and related models. Starting in early 2000s, DDDAS-based methods are used to enhance the modeling and instrumentation approaches discussed earlier to achieve improved efficiency in oil/gas discovery and recovery [Parashar 2005, 2006]. Similar approaches have also been pursued for intelligent fracking methods for exploration and recovery of oil \& gas.

\section{(G) Public Health Surveillance}

Syndromic surveillance using inpatient and ambulatory clinical care electronic health record (EHR) data as well as other non-traditional data sources is a relatively new practice in public health. As an example, the Epi-SPIRE system [Li, 2005] monitored the behavioral changes of a population (IBM T. J. Watson Research Center, which consists two sites during 2003 - Yorktown and Hawthorne, located in the Westchester County, New York) caused by the earliest stages of flu outbreak. Examples of such behavior changes include

- Increased number of people reporting (on a daily basis) that they are not feeling well among 400 volunteers

- increased absenteeism captured in the badge data for employee reporting to work,

- increased inquiries for medical information captured by the firewall web log,

- increased phone calls to make doctor appointments captured by the phone log,

- changes in eating/drinking habits captured by the cafeteria transaction data,

- increased coughing sound captured by the seminar recordings,

- increased traffic for leaving the building early captured by the video surveillance, and

- increased sewage generation as monitored by the facility.

The alerts generated from these data sources are compared to the insurance claims (as ground truth) from the Westchester County. It was demonstrated that the early warnings generated by some of the data sources (survey and phone in particular) provides leading indicators (up to 
three days) as compared to those derived from the clinical data sources, in a statistical significant way.

\section{SYSTEM IMPLICATIONS FOR FOG COMPUTING DEPLOYMENT}

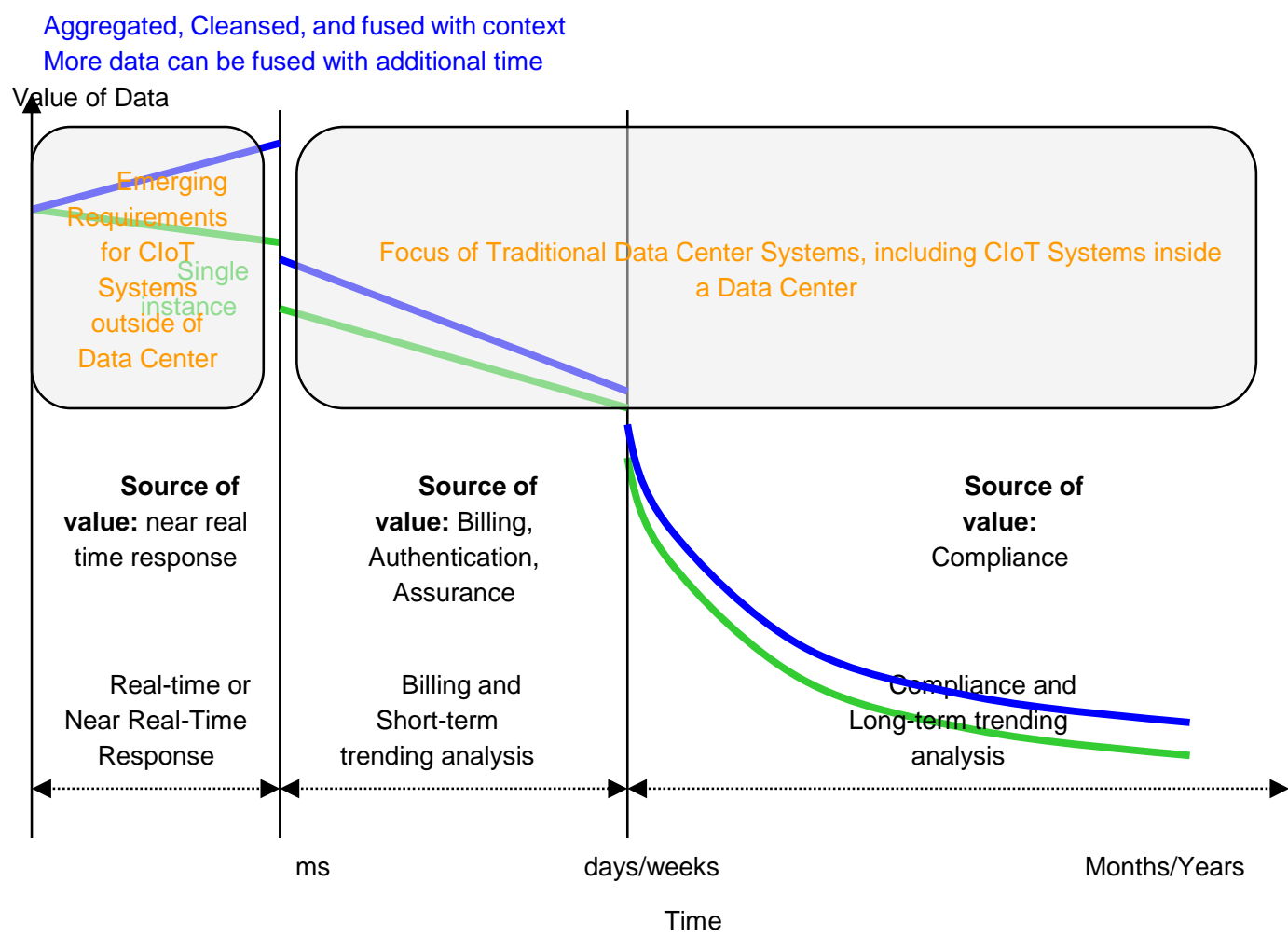

Figure 6: Providing aggregation, cleansing and fusion of Sensor Data closer to the Data Sources facilitates early response to critical events.

CIoT solutions often pose dynamic requirements for processing and analyzing data from sensors, derive decisions, and apply control through actuators. Figure 6 shows the value (potential business outcome) of the data for a CIoT solution as a function of time. The value of data is highest when real-time or near real-time decisions and proactive responses are possible. When multiple data sources are used as dynamic inputs into the behavior models, the value could be even higher as additional data can help to make the model more accurate, speed-up the model, reduce the uncertainty and contribute to improved accuracy for predicting future condition and evolution of the system. The value of these data may monotonically decline after the initial interval such as for example in the cases where data are mostly used 
for metering, billing, auditing, and long term trend analysis. Many applications do leverage "historical"/archival data in conjunction with those data acquired in real-time. As a result, system architectures optimized for CIoT solutions need to accommodate latency requirements and prioritize computation and communication resources in order to maximize the value that can be derived from the sensor data as well as the long term archival requirements to facilitate long term trend analysis.

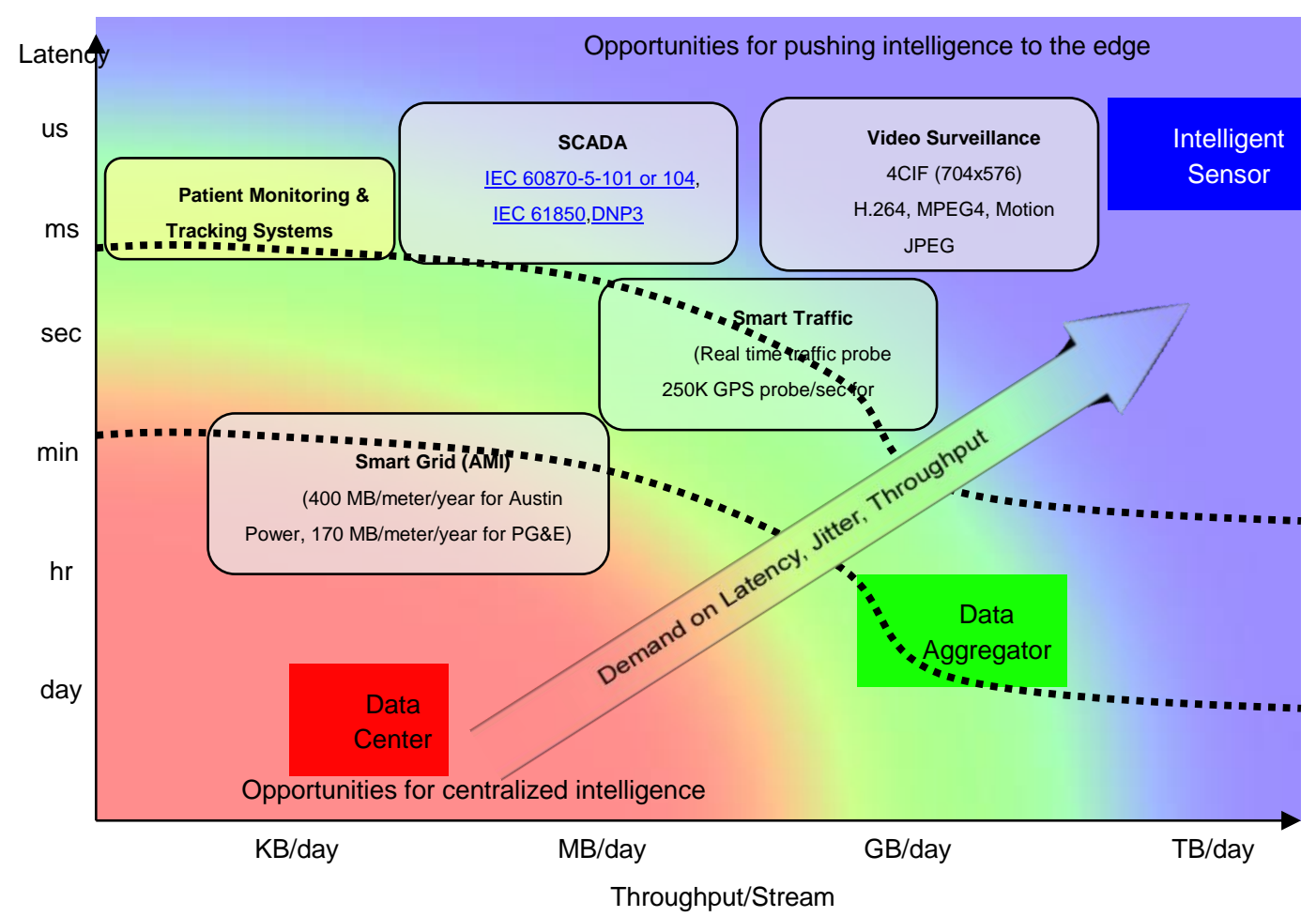

Figure 7: Latency and throughput requirements often drive the need for pushing intelligence to the edge.

Figure 7 shows the requirements coming from CIoT solutions in terms of throughput and latency. Video surveillance is at the forefront of these requirements in terms of throughput (3.2-26GB/day/stream). SCADA systems and health monitoring systems have very stringent latency requirements (on the order of microseconds to milliseconds). High throughput and/or low latency requirements often mandate moving some cognitive capabilities (e.g. some of the computation) to the edge (the sensors or controllers, or collections thereof) of the CIoT solution even though the primary analytic functions are still carried out in more powerful platforms, such as computing- and data-center(s), or other local or remote mid-range 
platforms. This trend suggests that deployment of CIoT solutions in traditional cloud (centralized) models is no longer adequate and necessitates the use of the nacent fog (or “edge") computing models.

Fog computing [Bonomi 2012] is an architecture that uses end-user clients or near-user edge of the network devices to carry out a substantial amount of storage, communication, control, configuration, measurement and management. Additional literature on fog computing addresses challenges to support such objectives [Cisco, 2015; Zaidi, 2014; Stojmenovic, 2014]. Compared to the initial notions of cloud computing, fog computing emphasizes

- proximity to end-users and client objectives,

- dense geographical distribution and local resource pooling,

- latency reduction for quality of service $(\mathrm{QoS})$ and edge analytics/stream mining.

Fog computing seeks to provide superior user experience and overall system redundancy in case of failure. It emphasizes the notions of information processing to generate the knowledge nearer the entity that needs it, regardless whether the entity is a human or an engineered device (or collections thereof). A CIoT solution, when either latency or throughput is critical (as indicated in the upper left, upper right, and lower right quadrants of Fig 7), could substantially benefit from exploiting fog computing concepts. It should be noted however that while the term fog computing concept was introduced in 2012, the concepts of distributed computing and distributed resource management - across a range of platforms, including computing at the edges (e.g. mobile devices, personal portable devices, sensors, etc) were ideas articulated many years before that [Darema, 1998, 2005], and will be discussed more in the next section. Also, to note that in the mid- to late-90s, Grid computing concepts [Foster, et.al. 1999] provided the impetus for moving from client-server computing to more general distributed computing (or metacomputing - a term coined in 1987 by Larry Smarr [Smarr1987]). Grid computing emphasized the coordination of multiple distributed computation, storage and communication resources. It has successfully demonstrated interoperability across multiple computational platforms used to support the runtime of a given application, as well as multiple applications executing concurrently. It should also be noted that the notion of virtualization, emphasized in cloud computing, was never precluded in the Grid concept. And, cloud computing has evolved over the years from a homogeneous centralized services concept to a 
more (geographically and otherwise) distributed and heterogeneous collections of platforms, with concomitant challenges of interoperability as were present in Grid computing. It is likely that Fog computing (or edge computing) may also evolve towards a hybrid and heterogeneous environment similar to cloud computing.

In summary, the fusion of the fog computing based deployment of the CIoT systems and solutions is likely to be heavily dependent on the latency and bandwidth requirement of the systems and solutions, and most likely be a hybrid and heterogeneous environment. Those system components (data acquisition, comprehension, and control actuation) residing outside of the lower left quadrants of Fig. 7 will likely leverage the low latency and high bandwidth offered by the fog computing. Those system components require the access of large amount of historical data and large amount of computation power (model construction, training and planning) is likely to stay in the cloud computing center, resulting a heterogeneous and hybrid environment.

\subsection{Systems Software and Cyber Infrastructure: Support and Tools}

There is a rapid convergence between the computational and instrumentation platforms for various CIoT solutions. Such integrated platforms are necessary to support DDDAS environments [Darema 1998; 2000; 2005; 2007], resulting in new systems software and cyberinfrastructure requirements, such as:

- Application programming models that support both high-end and real-time components of the application;

- Runtime support, which includes embedding the compiler into the runtime, allows mapping (and remapping) application components onto heterogeneous computing resources in optimized ways based on the architecture and capacity of the underlying computation, memory, communication and I/O resources. This support should span both high-end and the real-time environments, and be able to discover and match resources depending on the changing workload requirements of the executing application, such as in DDDAS-based application models. The software defined computing concept that originated from enabling simultaneous agility and optimal resource utilization in cloud 
computing could potentially play an increasingly important role for the runtime support. Software Defined Environments [Li, 2014], which include software defined networking, storage, and compute, decouple the control and management planes of these resources from the data planes so that they are no longer vertically integrated as in traditional compute, storage or switch systems and can be deployed anywhere within a computing environment.

- Application composition at runtime that supports dynamic selection of models based on (1) data streamed into the executing application, (2) application model interfaces, and (3) application knowledge based systems.

- Application data interfaces, which includes application-measurement interfaces and data models.

- Dynamic application execution support environments: System software is required to dynamically select application components and embody algorithms suitable for the specific solution approaches, cognizant of the varying underlying resources and application requirements.

- Dynamic computing and resource requirements: CIoT solutions require employing heterogeneous platform environments for data-collection, distributed high-performance simulations, and pre/post processing of data. Such environments require dynamic discovery of computational resources by system software to ensure fault tolerance and Quality of Service (QoS) in spite of changing workload conditions.

\section{OUTCOME BASED DISTRIBUTED ORCHESTRATION}

Outcome-based delivery model [Ng, 2013], in contrast to the input-based or output-based service delivery models, has generated substantial interests and adoption in multiple industries during the past decade, and is playing an increasingly important role in CIoT solutions.

In a service delivery model, the producers consume the input to produce output and result in outcome for the consumer: 
- Inputs: Include all of the resources (time and materials) used to produce and deliver the required output product or services. Examples of inputs include available full-time equivalents (FTEs), funds, time, equipment, raw materials, etc.

- Output: Include all of the products and services produced from the input. This could also involve business outputs, such as healthcare policy quote generation or enrollment processing, and IT outputs, such as test case creation and execution.

- Outcome: Measurable impacts delivered by providers of products or services that can be assessed objectively by services buyers. This could include business outcomes, such as improvement in the enrollment rate of a healthcare plan, or IT outcomes, such as reduced spending on quality assurance as a percentage of the IT budget.

Ideally, full provenance should be established so that all required inputs can be traced through the outputs and the outcomes. Business models in terms of how to price the products or services rendered have evolved based on input, output, outcome, or combinations of the above:

- Input-based delivery model: The producer charges the consumers based on the time and materials (and margin) needed to produce the products or services. The consumer often needs to assume most of the risk for the potential variation of the product or services received.

- Output-based delivery model: The producer charges the consumer based on the output products or services rendered. In this case, the producer assumes some amount of the risk arising from the variation of time and materials needed to develop the product and services, while the consumer also assumes certain amount of risk arising from the variation of the consequence resulting from the product or services.

- Outcome-based delivery model: The consumer is charged based on the actual business outcome from the products or services received. The producer needs to assume most or the entire risk for producing the desired outcome at the consumer.

There are multiple examples of such evolution. In the internet advertisement industry, there is a gradual evolution from pay per impression (prior to 2000), to pay per click (around 2000), to pay per action (around 2001), to revenue sharing (from 2001 onwards), and eventually to profit sharing (since 2010 in certain circumstances). In the healthcare industry, there is also a 
significant trend in recent years that the healthcare providers are being compensated from procedure based to patient health outcome based.

Outcome based delivery model has been considered as the most promising delivery model to integrate CIoT solutions into various industries. Model-based orchestration in CIoT solutions enables these solutions to continuously deliver the desired outcome. The outcomebased concept can be illustrated using those case studies in Section 4:

- Smart Grid solution: the business outcome for CIoT-based smart grid solutions refers to the cost saving for the customers; reduced maintenance cost for the operators; reduced or eliminated brown-out and black-out events; or reduced mean time to restore after a major weather event.

- Intelligent oil field solution: the business outcome refers to the reduced maintenance cost; or improved oil production throughput due to reduced down time.

- Intelligent traffic system: the business outcome refers to the reduced congestion, injuries and deaths due to accidents, and improved traffic flow.

- Software cybersecurity: the business outcome refers to the reduced or eliminated cyber perimeter penetration or data loss events.

- Cognitive syndromic surveillance: the business outcome refers to the reduced mortality and healthcare cost due to various infectious diseases.

Outcome-based orchestration of a CIoT solution shown in Fig. 2 requires orchestrating information and control flows through interconnected platforms. The fundamental challenge for a scalable CIoT solution is to address the centralized nature of orchestration. An outcomebased distributed orchestration framework, as shown in Fig. 8, is described in this section using outbreak risk prediction for Hantavirus Pulmonary Syndrome (HPS) [Glass 2002] as an example. HPS is a potentially fatal respiratory disease caused by infection with a rodent-born hantavirus. First reported in 1993, the case fatality rate was 50\%+ initially and has come down during recent years. The risk prediction model used the combination of remotely sensed data from satellite and elevation map on the ground to assess the likely population change of the rodents due to global vegetation change resulting from global climate change (e.g. El Niño followed by La Niña). Within this outcome-based framework, the orchestration is based on 
distributed observation, evaluation, and actuation of the real world. The key pillars that enable outcome-based orchestration are progressively represented data and models, as described in the following two subsections.

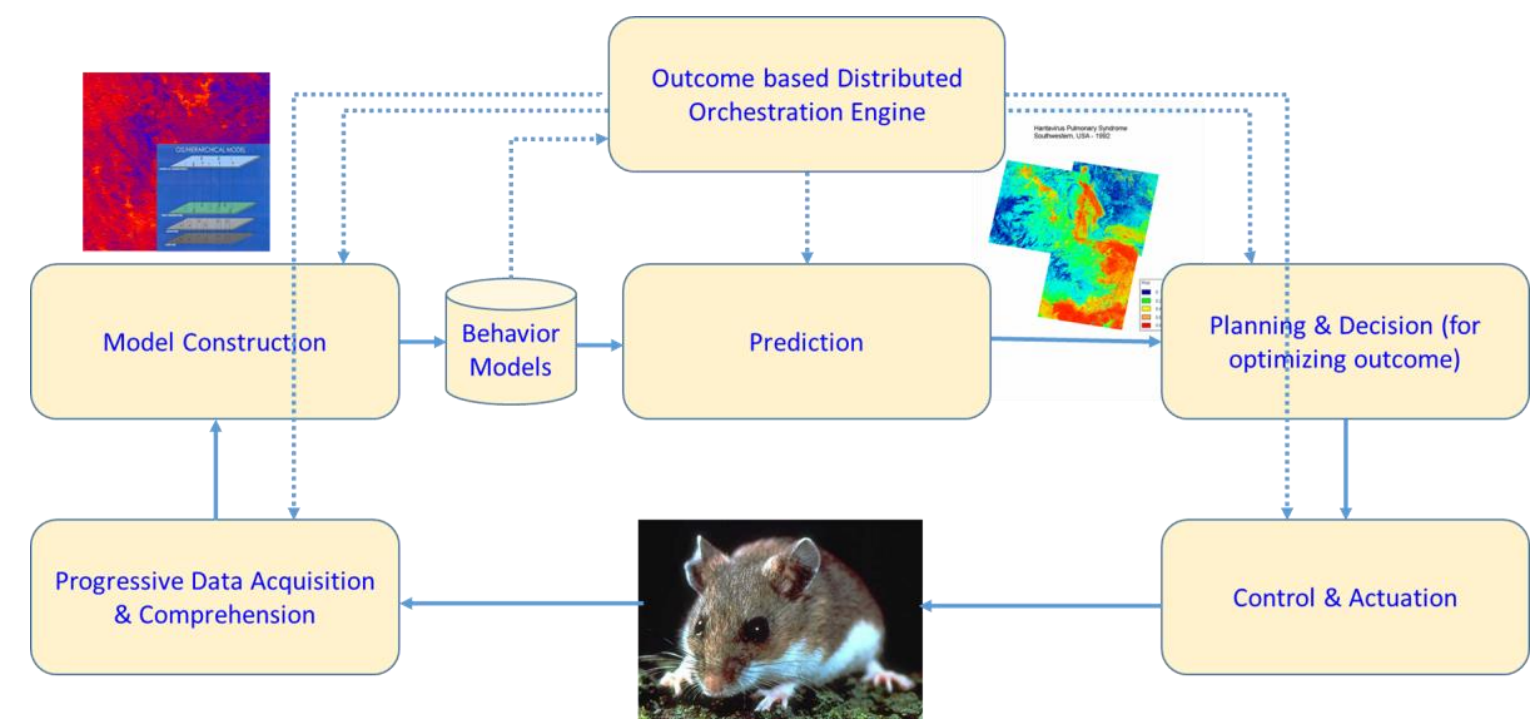

Figure 8: Distributed Outcome-based Orchestration framework for CIoT Solution using HPS outbreak risk prediction as an example.

\subsection{Progressive Data Representations}

Progressive $^{4}$ data representation is critical to progressive data acquisition, progressive transmission, and distributed model orchestration. Progressive representations enable the underlying phenomenon to be represented at multiple levels of abstraction (raw data, features, concepts), and multiple levels of fidelities, with trade-offs exist between fidelity (or accuracy) and data volume at each level.

\footnotetext{
${ }^{4}$ The term "progressive" is introduced here to denote time-dependent adaptivity in data and models, represented at multiple levels of abstraction, multiple levels of fidelity, and multiple modalities
} 
Two orthogonal dimensions - fidelity and abstraction - exist for generating progressive data representations. Multi-resolution representations, such as those based on discrete wavelet transformation (DWT), can be used to provide rough approximations of information at low resolutions (low data volumes), with more detailed views at higher resolutions. Most of the existing multi-resolution representation usually starts with the data already captured at its full resolution with homogeneous spatial and temporal sampling. In this paper, we propose to generalize such representations to include non-uniform acquisition, as it may be quite useful to selectively acquire higher resolutions only in selected "areas of interest". In contrast, multiple abstraction level representations relying on the fact that raw information can be processed into alternate formulations such as features and semantics that require lower data volumes at the expense of possible lower fidelity. For example, contours can be computed from a data array, allowing for very rapid identification of areas with low or high parameter values, but with a loss of accuracy.

\subsection{Progressive Models}

Progressive models are designed to enable real-time distributed orchestration of the models. Each instance of model executions in this distributed environment provides an estimate of the overall model predictions to be generated. In the case of data and measurement platforms, the distributed execution of the progressive models enables data acquisition density to be higher for regions of higher interests, and transmitted down sooner. Consequently, progressive model executions provide distributed prediction of optimal acquisition and transmission schedule within the data and measurement platforms. Progressive models usually involve the generation of coarse representations of the same model. Using the HPS risk prediction as an example [Glass, 2002], the linear regression model for predicting the risk of disease occurring at location $(\mathrm{x}, \mathrm{y})$ and time $\mathrm{t}$ is

$$
R(\mathrm{x}, \mathrm{y}, \mathrm{t})=\mathbf{a}_{1} \mathbf{x}_{1}(\mathrm{x}, \mathrm{y}, \mathrm{t})+\mathbf{a}_{2} \mathbf{x}_{2}(\mathrm{x}, \mathrm{y}, \mathrm{t})+\mathbf{a}_{3} \mathbf{x}_{3}(\mathrm{x}, \mathrm{y}, \mathrm{t})+\mathbf{a}_{4} R(\mathrm{x}, \mathrm{y}, \mathrm{t}-1)
$$

where $\mathbf{x}_{\mathrm{i}}(\mathrm{x}, \mathrm{y}, \mathrm{t})$ is the reading from the $i^{\text {th }}$ remote sensing instrument at location $(\mathrm{x}, \mathrm{y})$ and time $t$. If $\left|\mathbf{a}_{1}, \mathbf{a}_{2}\right| \gg>\left|\mathbf{a}_{3}, \mathbf{a}_{4}\right|$ then, a coarser representation of the model for $R(\mathrm{x}, \mathrm{y}, \mathrm{t})$ will be 


$$
R^{*}(\mathrm{x}, \mathrm{y}, \mathrm{t}) \sim \mathbf{a}_{1} \mathbf{x}_{1}(\mathrm{x}, \mathrm{y}, \mathrm{t})+\mathbf{a}_{2} \mathbf{x}_{2}(\mathrm{x}, \mathrm{y}, \mathrm{t})
$$

In general, the generation of progressively coarser representation of a model can be accomplished by analysing the relative contribution of each parameter to the overall model. This is similar to query planning issues in cost-based query optimization for object-relational databases. However, query planning usually rearrange the execution order so that operations achieving maximal filtering will be executed earlier. In contrast, outcome-based execution of progressive models will select operations that are most relevant to the final results (outcome) to be executed first.

\subsection{Outcome-based Orchestration}

Within the data and measurement platforms, the information will be progressively and proactively captured, processed and then transmitted to the modeling and orchestration platforms. The orchestration aims at achieving highest model prediction quality and business outcome by the modelling and orchestration platform at any given time, hence takes into account of the progressive data representation and model execution. This implies that the end results need to be superior at any time to all of the other possible data acquisition alternatives.

The value of progressive data acquisition lies in the identification of interested regions through progressive model processing, which can be executed anywhere, including the data and measurement platforms or the control platforms. Distributed processing at the edge of the network is necessary since it provides real-time feedback and control of the instruments, which is infeasible from a remote datacenter due to potentially long round-trip delay and transmission-processing lag.

Associated with the issue of prediction accuracy is the optimal balance of two-types of errors. The first type of errors is often referred to as "false alarms" or "false positives" when the low-risk areas are incorrectly identified as high-risk areas. The second type of errors is referred as "misses" or "false negatives" when the high-risk areas are identified as low-risk areas. In most cases, misses and false alarms cannot be simultaneously reduced [Daubie et al, 2002; Leonard et al., 2004; Chang, 2014; 2015]. Depending on the application requirements, 
an optimal allocation of misses and false alarms may be derived from model predictions to generate a progressive acquisition schedule.

The concept of progressive transmission may be formally defined as an optimization problem. Suppose $\mathrm{u}(t)$ is the utility function of the received data until time $t$. This utility function is a measure of the sum of the outbreak likelihood of received image regions until time $t$, with additional weighting factors taking into account population density, age distribution, environmental readiness, etc. Progressive transmission should order the regions in such a way that $\mathrm{u}(t)$ is maximized for any $t$. This implies small regions with the most contribution to the utility will be transmitted first. The intuition is that high-utility areas shall receive attention from decision functions as early as possible. Low-utility areas yield in the schedule because those areas may have less impact for the decision functions. In the simplest case with only one model parameter to acquire, a greedy scheme that orders the most valued to the least valued region generates the best schedule based upon the above criterion.

While discussions thus far only involve simple examples of single parameter acquisition, multiple parameters and more than one models with shared parameters may be constantly monitored by the CIoT solution. For example, mosquito-born diseases in public health surveillance solutions are often characterized by environmental factors like temperature and moisture. Depending on particular species of mosquitoes, temperature and moisture thresholds may vary. A satellite may be given several approximate models that share some parameters. In such case, progressive data acquisition and transmission scheduling become a sophisticated multi-parametric optimization problem. A parameter with strong influence on model prediction precision may be shared by three models while another parameter with weak influence on prediction precision is shared by five models. Both parameters' data acquisition compete for instruments, processing, storage, transmission capacity as well as other resources. However, their relative priority is not strict and is likely to vary depending on the acquired region.

It should be noted that specific behavior models are often difficult to be generalized as they are often tightly coupled with the data specific analytic environment. More general levels of abstraction of data and models are addressed through DDDAS/Infosymbiotics paradigm 
which provides a clear methodology of the value of dynamic integration of models and data in a feedback control loop.

\section{CONCLUSIONS}

The introduction of pervasive and ubiquitous instrumentation leads to unprecedented realtime visibility in many application areas such as the power grid, traffic, transportation, water, and oil \& gas areas. Interconnecting and harnessing those distinct natural, engineered, people, and business worlds through ubiquitous instrumentation, even though still in its embryonic stage, is rapidly progressing and has started demonstrating the potential to engender capabilities for an environment that is greener, efficient, safer, and more comfortable.

In this paper, we presented an outcome-based framework for orchestrating both information and control flows within CIoT systems and solutions based on interconnected cognitive models of these environments. In contrast to the traditional prescriptive orchestration approach where a detailed script is provided, the proposed approach specifies the desired outcome and orchestrates information and control flows, using the behavior model of the CIoT system. The orchestration is executed based on progressive data (dynamic data) acquisition and representation, and schedules the operations for the CIoT systems and solutions progressively using a distributed model. This framework is validated through use cases such as load shedding and disaster recovery for smart grid, structural health monitoring for intelligent aerial platforms, public health surveillance, and cybersecurity. It has become increasingly crucial that cognitive representations of these distinct worlds (a.k.a. models, dynamically integrated with instrumentation, such as in DDDAS-based methods) need to be created as a pre-requisite for assessing and intelligently managing the complexity, maneuvering through uncertain environments and eventually achieving the optimized outcomes. We expect the proposed outcome-based framework will contribute to the development of emerging services and analytics for Cognitive Internet of Things (CIoT) based solutions, as well as serving as the foundation for enterprises using outcome-based business models for their products and services.

The primary challenges in the next few years are likely to closely track the progress made in the cognitive computing area in terms of encoding domain specific knowledge 
in specific knowledge representations, automatic knowledge-based construction $(\mathrm{AKBC})$, integrating knowledge driven and data driven approaches together on common behavior level models. Most importantly, the focus is likely to be centered on the linkage between the business outcome and the outcome accomplished by the CIoT systems and solutions.

\section{ACKNOWLEDGEMENT}

This work was conducted while C.-S. Li was with IBM T. J. Watson Research Center. The authors would like to acknowledge the discussions with Kevin McAuliffe, Art Ciccolo, Nagui Halim, Josyula R. Rao, John R. Smith, Brenda Dietrich, Anant Jhingran and Charles Lickel during the development of the original concept. The authors also like to acknowledge Ms. Annie An Dongmei for proofreading the manuscript.

\section{REFERENCES}

Abarbanel, B., and McNeely, W. Flythru the boeing 777. ACM SIGGRAPH 96 Visual Proceedings: The art and interdisciplinary programs of SIGGRAPH'96. ACM, 1996.

Akcelik, Volkan Jacobo Bielak, George Biros, Ioannis Epanomeritakis, Omar Ghattas, Loukas F. Kallivokas, and Eui Joong Kim, A framework for online inversion-based 3D site characterization, in Computational Science - ICCS 2004: 4th International Conference, Krakow, Poland, June 6-9, 2004

Akcelik, V. , G. Biros, A. Draganescu, O. Ghattas, J. Hill, and B. van Bloemen Waanders, Inversion of airborne contaminants in a regional model, in Computational Science ICCS 2006: 6th International Conference, Reading, UK, May 28-31, 2006,

Ali, A. A., Goel, A., Ridley, A. J., \& Bernstein, D. S. (2015). Retrospective-Cost-Based Adaptive Input and State Estimation for the Ionosphere-Thermosphere. Journal of Aerospace Information Systems, 1-17.

Allaire, D., Kordonowy, D., Lecerf, M., Mainini, L., \& Willcox, K. (2014). Multifidelity DDDAS methods with application to a self-aware aerospace vehicle. Procedia Computer Science, 29, 1182-1192.

Atzori, L., Iera, A., \& Morabito, G. (2010). The internet of things: A survey. Computer networks, 54(15), 2787-2805.

Babaoglu, O., Marzolla, M., \& Tamburini, M. (2012, March). Design and implementation of a P2P Cloud system. In ACM Proceedings of the 27th Annual ACM Symposium on Applied Computing (pp. 412-417).

Bates, P. C. (1995). Debugging heterogeneous distributed systems using event-based models of behavior. ACM Transactions on Computer Systems (TOCS), 13(1), 1-31.

Bazilevs, Y., Marsden, A. L., di Scalea, F. L., Majumdar, A., \& Tatineni, M. (2012). Toward a computational steering framework for large-scale composite structures based on continually and dynamically injected sensor data.Procedia Computer Science, 9, 1149-1158.

Bonomi, F., Milito, R., Zhu, J., \& Addepalli, S. (2012, August). Fog computing and its role in the internet of things. In ACM Proceedings of the first edition of the MCC workshop on Mobile cloud computing (pp. 1316).

Boyer, S. A. (2009). SCADA: supervisory control and data acquisition. International Society of Automation.

Brotzge, J., V. Chandresakar, K. Droegemeier, J. Kurose, D. McLaughlin, B. Philips, M. Preston, and S. Sekelsky, Distributed Collaborative Adaptive Sensing for Hazardous Weather Detection, Tracking, and Predicting, ICCS05Proceedings (2004) pp. 670-677.

Cannon, David (2011). ITIL Service Strategy 2011 Edition. The Stationery Office.

Carmichael, G. R., Daescu, D. N., Sandu, A., \& Chai, T. (2003). Computational aspects of chemical data assimilation into atmospheric models. In Computational Science-ICCS 2003 (pp. 269-278). Springer Berlin Heidelberg. 
Celik, N., Lee, S., Vasudevan, K., \& Son, Y. J. (2010). DDDAS-based multi-fidelity simulation framework for supply chain systems. IIE Transactions,42(5), 325-341.

Celik, Nurcin, et al. "DDDAMS-based Real-time Assessment and Control of Electric-Microgrids, ICCS2011 Proceedings (2011)

Chang, V. (2014). The business intelligence as a service in the cloud. Future Generation Computer Systems, $37,512-534$.

Chang, V. (2015). A proposed cloud computing business framework. Nova Science Publisher.

Chang, V. (2017 a) A Cybernetics Social Cloud. Journal of Systems and Software, 124, 195-211.

Chang, V. (2017). Towards data analysis for weather cloud computing. Knowledge-Based Systems, 127, 29-45.

Chen, W. S. E., Huang, C. F., \& Huang, M. J. (2016). iSDS: a self-configurable software-defined storage system for enterprise. Enterprise Information Systems, 1-22.

Cheng, X., and Dang, G The P2P communication technology research based on Internet of things. Advanced Research and Technology in Industry Applications (WARTIA), 2014 IEEE Workshop on. IEEE, 2014.

Cheng, Y., Tao, F., Xu, L., \& Zhao, D. (2016). Advanced manufacturing systems: supply-demand matching of manufacturing resource based on complex networks and Internet of Things. Enterprise Information Systems, 1-18.

Chesbrough, H. (2010). Business model innovation: opportunities and barriers. Long range planning 43.2 (2010): 354-363.

Cisco Fog Computing (2015): http://www.cisco.com/c/dam/en_us/solutions/trends/iot/docs/computingsolutions.pdf

Cloud Computing: http://www.cmswire.com/cms/information-management/cloud-computing-back-to-thefuture-020101.ph

Darema, F., The Next Generation Program (1998); http://www.nsf.gov/pubs/1999/nsf998/nsf998.htm

Darema, F., The Next Generation Software Program, http://www.nsf.gov/pubs/2000/nsf00134/nsf00134.htm; and International Journal of Parallel Programming, June 2005, Volume 33, Issue 2, pp 73-79

Darema, F. , Grid Computing and Beyond: The Context of Dynamic Data Driven Applications Systems, Proceedings of the IEEE, Special Issue on Grid Computing, March 2005 (invited paper)

Darema, F., Dynamic Data Driven Applications Systems (DDDAS). NSF Workshop 2000, and 2006 and 2010 Workshopes (www.1dddas.org); Dynamic Data Driven Applications Systems (DDDAS): New Capabilities for Application Simulations and Measurements; ICCS05Proceedings (2005)

Darema, F. The Next Generation Software Workshop - IPDPS'07, Proceedings of IPDPS 2007

Daubie, M., Levecq, P., \& Meskens, N. (2002). A comparison of the rough sets and recursive partitioning induction approaches: An application to commercial loans. International Transactions in Operational Research, 9(5), 681-694.

Ding, Y., Byon, E., Park, C., Tang, J., Lu, Y., \& Wang, X. (2007). Dynamic data-driven fault diagnosis of wind turbine systems. In Computational Science-ICCS 2007 (pp. 1197-1204). Springer Berlin Heidelberg.

Farzaneh A., Ovatman, T., Quality of Service Trade-offs Between Central Data Centers and Nano Data Centers, the 5th International Conference on Cloud Computing and Services Science, 2015 Lisbon, Portugal.

Foster, I.; Kesselma, C.n (1999). The Grid: Blueprint for a New Computing Infrastructure. Morgan Kaufmann Publishers. ISBN 1-55860-475-8.

Gallagher, Sean. How IBM's Deep Thunder delivers hyper-local forecasts 3-1/2 days out. Ars Technica (March 14, 2012).

Gazis, Vangelis, et al. "Wireless Sensor Networking, Automation Technologies and Machine to Machine Developments on the Path to the Internet of Things." Informatics (PCI), 2012 16th Panhellenic Conference on. IEEE, 2012.

Glass, G.E., Yates, T.L., Fine, J.B., Shields, T.M., Kendall, J.B., Hope, A.G., Parmenter, C.A., Peters, C.J., Ksiazek, T.G., Li, C.S. and Patz, J.A., 2002. Satellite imagery characterizes local animal reservoir populations of Sin Nombre virus in the southwestern United States. Proceedings of the National Academy of Sciences, 99(26), pp.16817-16822.

Gill, P., Jain, N., \& Nagappan, N. (2011, August). Understanding network failures in data centers: measurement, analysis, and implications. In ACM SIGCOMM Computer Communication Review (Vol. 41, No. 4, pp. 350-361). 
Granados, Jose, et al. Towards energy-efficient HealthCare: An Internet-of-Things architecture using intelligent gateways. Wireless Mobile Communication and Healthcare (Mobihealth), 2014 EAI 4th International Conference on. IEEE, 2014.

Gubbi, J., Buyya, R., Marusic, S., \& Palaniswami, M. (2013). Internet of Things (IoT): A vision, architectural elements, and future directions. Future Generation Computer Systems 29(7): 1645-1660.

Hariri, Salim, Cihan Tunc, Pratik Satam , Firas Al-Moualem, and Erik Blasch, "DDDAS-based Resilient Cyber Battle Management Services (d-RCBMS)," proceedings of the HiPC 2015 DDDAS Workshop, December 16, 2015, Bengaluru, India.

Hoffmann, C. et al "DDDAS For Autonomic Interconnected Systems: The National Energy Infrastructure", ICCS2007 Proceedings (2007)

Karagiannis, V., Chatzimisios, P., Vazquez-Gallego, F., \& Alonso-Zarate, J. (2015). A survey on application layer protocols for the internet of things.Transaction on IoT and Cloud Computing, 3(1), 11-17.

Korobenko, A., Hsu, M. C., Akkerman, I., Tippmann, J., \& Bazilevs, Y. (2013). Structural mechanics modeling and FSI simulation of wind turbines.Mathematical Models and Methods in Applied Sciences, 23(02), 249272.

Letz, S., Lewis, W. J., Licker, S. M., Mallar, H., McCain, E. C., Roesner, W., ... \& Winkelmann, R. (2009). Functional verification of the IBM system z10 processor chipset.

Li, Chung-Sheng. "Real-time event driven architecture for activity monitoring and early warning." Emerging Information Technology Conference, 2005.. IEEE, 2005.

Li, C.-S., B. L. Brech, S. Crowder, D. M. Dias, H. Franke, M. Hogstrom, D. Lindquist, G. Pacifici, S. Pappe, B. Rajaraman, J. Rao, R. P. Ratnaparkhi, R. A. Smith and M. D. Williams. Software defined environments: An introduction. In IBM Journal of Research and Development Vol. 58 No. 2/3 pp. 1-11, March/May, 2014.

Leonard, M., Graham, S., \& Bonacum, D. (2004). The human factor: the critical importance of effective teamwork and communication in providing safe care. Quality and Safety in Health Care, 13(suppl 1), i85i90.

McCalley, J., Honavar, V., Ryan, S., Meeker, W., Qiao, D., Roberts, R., ... \& Hong, Y. (2007). Integrated decision algorithms for auto-steered electric transmission system asset management. In Computational Science-ICCS 2007 (pp. 1066-1073). Springer Berlin Heidelberg.

Mendez, V., et al. Powering Distributed Applications with DIRAC Engine. Proceedings of the symposium. Vol. 1. 2014.

Min, H., and Zhou, G. Supply chain modeling: past, present and future. Computers \& industrial engineering 43.1 (2002): 231-249.

Muñoz, V. M., Ramo, A. C., Albor, V. F., Diaz, R. G., \& Arévalo, G. M. (2013). Rafhyc: An architecture for constructing resilient services on federated hybrid clouds. Journal of Grid Computing, 11(4), 753-770.

$\mathrm{Ng}$, Irene CL, David Xin Ding, and Nick Yip. "Outcome-based contracts as new business model: The role of partnership and value-driven relational assets." Industrial Marketing Management 42.5 (2013): 730-743.

Ning, H., Belanger, D. G., Xia, Y., Piuri, V., \& Zomaya, A. Y. (2015). Guest Editorial Special Issue on Big Data Analytics and Management in Internet of Things. Internet of Things Journal, IEEE, 2(4), 265-267.

Parashar, M., Matossian, V., Bangerth, W., Klie, H., Rutt, B., Kurc, T., Catalyurek, U., Saltz, J., and Wheeler, M. F. (2006) Towards Dynamic Data-Driven Optimization of Oil Well Placement, Lecture Notes in Computer Science (LNCS) 3515, pp. 656-663, 2005

Parashar, M., Matossian, V., Klie, H., Thomas, S., Wheeler, M. F. B., Kurc, T., Saltz, J., and Versteeg, R. (2006) Towards Dynamic Data-Driven Management of the Ruby Gulch Waste Repository, Lecture Notes in Computer Science (LNCS) 3993, pp. pp 384-392, 2006.

Patra, A. K., Bursik, M., Dehn, J., Jones, M., Madankan, R., Morton, D., ... \& Singla, P. (2013). Challenges in Developing DDDAS based Methodology for Volcanic Ash Hazard Analysis-Effect of Numerical Weather Prediction Variability and Parameter Estimation. Procedia Computer Science, 18, 1871-1880.

Peng, L., Silic, M., \& Mohseni, K. (2015). A DDDAS plume monitoring system with reduced Kalman filter, Procedia Computer Science, Vol 51, 2015, pp 2533-2542

Peng, L. and Mohseni, K., Symplectic model reduction of Hamiltonian systems, SIAM Journal on Scientific Computing, 38(1), A1-A27, 2016

Plale, B., Gannon, D., Reed, D., Graves, S., Droegemeier, K., Wilhelmson, B., \& Ramamurthy, M. (2005, May), Towards Dynamically Adaptive Weather Analysis and Forecasting in LEAD. In International Conference on Computational Science (pp. 624-631). Springer, Berlin, Heidelberg. 
Prudencio, E.E., P. T. Bauman, D. Faghihi, and J. T. Oden, K. Ravi-Chandar, and S. V. Williams, A Dynamic Data Driven Application System for Real-time Monitoring of Stochastic Damage, ICCS05Proceedings (2013) pp. 2056-2065.

Richardson, S., Thomson, A., Best, N., \& Elliott, P. (2004). Interpreting posterior relative risk estimates in disease-mapping studies. Environmental Health Perspectives, 1016-1025.

Riggins, F. J., \& Wamba, S. F. (2015, January). Research directions on the adoption, usage, and impact of the internet of things through the use of big data analytics. In System Sciences (HICSS), 2015 48th Hawaii International Conference on (pp. 1531-1540). IEEE.

Jiang, R. and Sun, D. Architecture Design of the Internet of Things based on Cloud Computing. Measuring Technology and Mechatronics Automation (ICMTMA), 2015 Seventh International Conference on. IEEE, 2015.

Rao, V., \& Sandu, A. (2014). A posteriori error estimates for DDDAS inference problems. Procedia Computer Science, 29, 1256-1265.

Shang, X., Zhang, R., Zhu, X., \& Zhou, Q. (2016). Design theory, modelling and the application for the Internet of Things service. Enterprise Information Systems, 10(3), 249-267.

Smarr, L. - http://archive.ncsa.illinois.edu/Cyberia/MetaComp/MetaHistory.html

Stojmenovic, I. and Wen Sh., The Fog Computing Paradigm: Scenarios and Security Issues; Proceedings of the 2014 Federated Conference on Computer Science and Information Systems, ACSIS, Vol.2, pp 1-8, 2014

Subashini, S., \& Kavitha, V. (2011). A survey on security issues in service delivery models of cloud computing. Journal of network and computer applications, 34(1), 1-11.

Ubeda Garcia, Mario, et al. "Integration of Cloud resources in the LHCb Distributed Computing." J. Phys.: Conf. Ser.. Vol. 513. 2014.

Sundmaeker, A. Bassi, et al., Internet of Things: Global Technological and Societal Trends, 1, 9-52.

Wang, C., Wang, Q., Ren, K., \& Lou, W. (2010, March). Privacy-preserving public auditing for data storage security in cloud computing. In IEEE INFOCOM, 2010 Proceedings IEEE (pp. 1-9).

Wang, X. V., \& Wang, L. (2016). A cloud-based production system for information and service integration: an internet of things case study on waste electronics. Enterprise Information Systems, 1-17.

Welbourne, Evan, et al. "Building the internet of things using RFID: the RFID ecosystem experience." Internet Computing, IEEE 13.3 (2009): 48-55.

Wu, Q., Ding, G., Xu, Y., Feng, S., Du, Z., Wang, J., \& Long, K. (2014). Cognitive internet of things: a new paradigm beyond connection. Internet of Things Journal, IEEE, 1(2), 129-143.

Zaidi, S. A. R., Shakir, M. Z., Imran, M. A., Ghogho, M., Vasilakos, A., Qaraqe, K., \& McLernon, D. (2014, October). Cognitive internet of things: A unified perspective. In International Internet of Things Summit (pp. 201-210). Springer, Cham.

Zaslavsky, A., Perera, C., \& Georgakopoulos, D. (2013). Sensing as a service and big data. arXiv preprint arXiv:1301.0159.

Zhai, C., Zou, Z., Zhou, Q., Mao, J., Chen, Q., Tenhunen, H., ... \& Xu, L. (2017). A 2.4-GHz ISM RF and UWB hybrid RFID real-time locating system for industrial enterprise Internet of Things. Enterprise Information Systems, 11(6), 909-926.

Zhou, L., \& Chao, H. C. (2011). Multimedia traffic security architecture for the internet of things. Network, IEEE, 25(3), 35-40. 